Pacific Journal of Mathematics

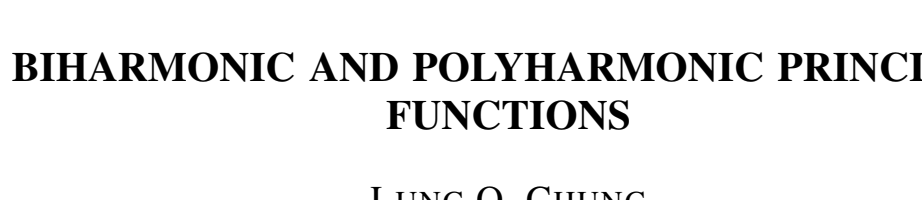




\title{
BIHARMONIC AND POLYHARMONIC PRINCIPAL FUNCTIONS
}

\author{
L. O. ChUnG
}

The biharmonic principal function problem is the construction of a biharmonic function in a space which "imitates" the behavior of a given singularity function. In this paper we first define the notion of a biharmonic operator which clarifies the modes of "imitation." We then prove the existence and uniqueness theorem of the biharmonic principal function. The theory is a generalization of the harmonic principal functions to the larger family of biharmonic functions. An indication of its application as well as its further generalization to polyharmonic functions is also given.

The theory of principal functions plays an important role in the study of harmonic functions in that it allows for the global construction of harmonic functions with a great variety of singularity behaviors. See Ahlfors-Sario [1] or Rodin-Sario [3] for a comprehensive treatment of this theory and many of its applications. Since the study of biharmonic and polyharmonic functions draws heavily from the experience of harmonic theory, it has been felt that a theory of biharmonic or polyharmonic principal functions would be desirable. Except for some results in the thesis of Rader [2] which constructs some interesting special examples, a general theory is still in the waiting. In this paper, we will present another step toward such a theory in first defining the basic concept of biharmonic operator and then proving the existence and uniqueness of biharmonic principal functions, one of the three main theorems of the theory. Our paper is self contained except for a proof of Sario's $q$-lemma which can be found in [1] or [3]. An indication of generalizing the results to polyharmonic functions is given at the end.

In $\S 1$, after a review of notations, we prove an alternating lemma which is the main technical tool of our theory. From this, the main existence and uniqueness theorem of Sario et al for the harmonic theory follows rather easily.

In $\S 2$, we define the concepts of a biharmonic operator which is basic for our theory. Examples and simple properties of this operator are given. Next in $\S 3$, we prove the existence and uniqueness theorem for biharmonic principal functions. Some applications are then given in $\S 4$ which includes the construction of biharmonic functions with various singularity properties, e.g., the classical singularity problem of closed manifolds, the biharmonic 
Green's function $\gamma$. Finally, in $\S 5$ we make some remarks about the generalization to polyharmonic functions as well as some open problems.

1. Let $R$ be a Riemannian manifold, compact or noncompact, and $\Delta=d \delta+\delta d$ the Laplace-Beltrami operator. A function $u(x)$ is called harmonic, quasiharmonic and biharmonic if $\Delta u=0, \Delta u=c$ with $c$ a constant and $\Delta^{2} u=\Delta \Delta u=0$ respectively. We will denote them, in symbols, by $H(R), Q(R)$ and $H^{2}(R)$ in that order. A quasiharmonic function $q$ such that $\Delta q=1$ is called a normalized quasiharmonic function. This terminology is slightly different from the one used by other workers in the field in that, in our usage, $\boldsymbol{R} \subseteq$ $H(R) \subseteq Q(R) \subseteq H^{2}(R)$ form a sequence of increasing vector spaces under inclusion where $\boldsymbol{R}$ is the set of real numbers.

We also consider manifolds with boundaries. Suppose that $A$ is a compact or noncompact manifold with boundary $\partial A$.. The above symbols $H(A), Q(A), H^{2}(A)$ will denote the family of harmonic, quasiharmonic and biharmonic functions in the interior of $A$ and continuous through the boundary $\partial A$. The following is the famous $q$-lemma of Sario whose proof can be found in [1] or [3]:

Sario's q-lemma. Let $\alpha$ be a compact subset of the interior of a Riemannian manifold $R$. Then there exists a positive constant $q<1$ such that for all harmonic functions $u$ on $R$ which change signs on $\alpha$, we have

$$
q \inf _{R} u \leqq u \mid \alpha \leqq q \sup _{R} u
$$

Before we prove the technical alternating lemma, we recall Sario's notion of normal operator, here we rename it harmonic operator. As above, let $A$ be a compact or noncompact Riemannian manifold with a compact boundary $\partial A$. A linear transformation $L: C(\partial A) \rightarrow H(A)$ is called a harmonic operator if it satisfies the following conditions: (1) $(L f) \mid \partial A=f, \quad(2) \min _{\partial A} f \leqq L f \leqq \max _{\partial A} f$ and (3) the flux condition: $\int_{\beta}^{*} d L f=0$ holds for any $\beta$ homologous to $\alpha$.

Alternating Lemma. Let $A, \Omega$ be submanifolds of $R$ such that

1. $\operatorname{dim} A=\operatorname{dim} \Omega=\operatorname{dim} R$,

2. A has a compact relative boundary $\partial A, \Omega$ has a compact relative boundary $\partial A$, and $\partial A \cap \partial \Omega=\phi$,

3. $A \cap \Omega$ is a nonempty open set, $\overline{A \cap \Omega}$ has boundary $\partial A \cup \partial \Omega$,

4. $L: C(\partial A) \rightarrow H(A)$ and $K: C(\partial \Omega) \rightarrow H(\Omega)$ are harmonic operators 
with $\max K(f)$, $\min K(f) \notin \operatorname{int}(\Omega)$ for any nonconstant $f$,

5. $w$ is a harmonic function on $A \cap \Omega$ continuous in $\overline{A \cap \Omega}$.

Then there are $u \in C(\partial A)$ and $v \in C(\partial \Omega)$ and a constant $k$ such that

$$
w-(L u+K v)=-k \omega,
$$

where $\omega$ is the harmonic function in $A \cap \Omega$ with boundary data 0 on $\partial A$ and 1 on $\partial \Omega$.

Further the constant $k=0$ if and only if the flux condition $\int_{\beta}^{*} d w=0$ holds for any $\beta$ homologous to $\alpha=\partial A$.

Proof. Consider the sequence

$$
(K L)^{n}(w-K w), \quad n=0,1,2, \cdots .
$$

The ranges range $(w-K w) \supset$ range $(K L)(w-K w) \supset$ range $(K L)^{2}(w-$ $K w) \supset \cdots$ form a decreasing sequence of closed sets by the definition of harmonic operators. Also, the maximum condition 4 of the hypothesis for $K$ implies that $\max (K L)^{n}(w-K w)>\max (K L)^{n+1}(w-$ $K w)$ and $\min (K L)^{n}(w-K w)<\min (K L)^{n+1}(w-K w)$. Therefore, there is a number $k \in \cap_{n}$ range $(K L)^{n}(w-K w)$. Form a new sequence

$$
(K L)^{n}(w-K w)-k=(K L)^{n}(w-k-K w), \quad n=0,1,2, \cdots .
$$

Each one of this sequence changes signs. Similarly $L(K L)^{n}(w-k-$ $K w)$ changes signs for each $n$ also. As a consequence, $(K L)^{n}(w-$ $k-K w)$ changes signs on the closed set $\partial A$ for each $n$. By the $q$-lemma, there is a $q \in(0,1)$ such that

$$
\begin{gathered}
q \inf _{\Omega}(K L)^{n}(w-k-K w) \leqq(K L)^{n}(w-k-K w) \mid \partial A \\
\leqq q \sup _{\Omega}(K L)^{n}(w-k-K w) .
\end{gathered}
$$

Since $\inf _{\partial A}(K L)^{n}(w-k-K w) \leqq(K L)^{n+1}(w-k-K w) \leqq \sup _{\partial A}(K L)^{n}(w-$ $k-K w)$, we have $q^{2} \inf _{\Omega}(K L)^{n}(w-k-K w) \leqq(K L)^{n+1}(w-k-K w) \mid \partial A \leqq$ $q^{2} \sup _{\Omega}(K L)^{n}(w-k-K w)$. Continuing, we have

$$
\begin{gathered}
q^{n} \inf _{\Omega}(K L)(w-k-K w) \leqq(K L)^{n}(w-k-K w) \mid \partial A \\
\leqq q^{n} \sup _{\Omega}(K L)(w-k-K w) .
\end{gathered}
$$

Thus, the series

$$
\sum_{n=1}^{\infty}(K L)^{n}(w-k-K w)
$$

converges to a harmonic function on $\Omega$. Similarly, the series 
$\sum_{n=1}^{\infty} L(K L)^{n}(w-k-K w)$ also converges to a harmonic function on $A$.

Let $v=k+K w-\sum_{n=1}^{\infty}(K L)^{n}(w-k-K w)$ and $u=\sum_{n=0}^{\infty} L(K L)^{n}(w-$ $k-K w)$. Consider the harmonic function $w-(K v+L u)=w-k-$ $K w+\sum_{n=1}^{\infty}(K L)^{n}(w-k-K w)-\sum_{n=0}^{\infty} L(K L)^{n}(w-k-K w)$. To see that it is $-k \omega$, we have to check that it has the desired boundary values. Operating with $K$, we see $K(w-(K v+L u))=K w-(K v+$ $K L u)=-k$; while operating with $L$, we have $L(w-(K v+L u))=$ $L w-(L K v+L u)=0$. Thus it has the boundary values $-k$ at $\partial \Omega$ and 0 at $\partial A$, as desired.

Finally, we will check the flux condition. Clearly,

$$
-\int_{\beta} * d k \omega=\int_{\beta} * d(w-(L u+K v))=\int_{\beta} * d w .
$$

Since $\int_{\beta} * d \omega \neq 0$, we conclude that $\int_{\beta} * d w=0$ if and only if $k=0$.

In many applications of the above alternating lemma, $R$ will be an open Riemannian manifold, $A$ will be a boundary neighborhood, $\Omega$ will be a regular submanifold of $R, K$ will be the Dirichlet operator on $\Omega$. Indeed, that is what our notations will be if not specified otherwise. We also use $\beta$ to denote a chain homologous to $\alpha$. As an application of this lemma, we give a new proof of the Main Existence and Uniqueness Theorem of the harmonic theory due to Sario et al, [1] and [3].

Theorem. Let $s$ be harmonic in $A$. Then the necessary and sufficient condition for the existence of a harmonic function $p$ on the entire $R$ such that

$$
p-s=L(p-s)
$$

is that $s$ satisfies the flux condition:

$$
\int_{\beta} * d s=0
$$

The functions $p$ is unique up to an additive constant.

Proof. Let $s$ be given. Let $\Omega$ be any regular subregion such that the alternating lemma applies. Then there are $u$ and $v$ such that $s-(L u+K v)=0$ by the lemma. Thus let $p=s-L u$ on $A$ and $p=K v$ on $\Omega$. Clearly, $p$ is the required harmonic function. This proves the sufficiency part. The necessity part is obvious.

The function $s$ that satisfies the above flux condition is called a singularity function, and the function $p$ is called a principal function of $s$ for the operator $L$. Intuitively, $p$ can be said to "imitate" 
the behavior of $s$ with respect to $L$. Our main objective is to extend the above theorem to the larger family of biharmonic functions.

2. To achieve our goal, we first define the concept biharmonic operator. A linear transformation $L: C(\partial A) \times C(\partial A) \rightarrow H^{2}(A)$ is called a biharmonic operator if it satisfies the following five conditions:

(1) $u=L f=L\left(f_{1}, f_{2}\right)$ is a bounded biharmonic function, i.e., $-\infty<\inf u \leqq \sup u<\infty$;

(2) $u\left|\partial A=f_{1}, \Delta u\right| \partial A=f_{2}$;

(3) $L(\cdot, 0)$ is a harmonic operator of the first variable;

(4) $\Delta L(0, \cdot)$ is a harmonic operator of the second variable;

(5) $L(0, f) \geqq 0$ if $f \geqq 0$.

We give some simple properties and examples of biharmonic operators.

For any $\alpha, A, f=\left(f_{1}, f_{2}\right)$ and any $L: C(\partial A) \times C(\partial A) \rightarrow H^{2}(A)$, we have the decomposition $L\left(f_{1}, f_{2}\right)=L\left(f_{1}, 0\right)+L\left(0, f_{2}\right)$. The first term of the above decomposition may be called the harmonic part and the second term the potential part of $L\left(f_{1}, f_{2}\right)$. To define $L$, we only need to define its harmonic part and potential part.

The following lemma on quasiharmonic functions is needed later.

Lemma. Let $A, \Omega$ be submanifolds of $R$ as in the alternating lemma. Let $L: C(\partial A) \times C(\partial A) \rightarrow H^{2}(A)$ and $K: C(\partial \Omega) \times C(\partial \Omega) \rightarrow H^{2}(A)$ be two biharmonic operators. Let $K(0,1)>0$ outside of $\partial \Omega$, and let $w=L(0,1)-K(0,1)$. Then there are $u, v$ and constant $k \neq 0$ such that

$$
w-(L(u, 0)+K(v, 0))=-k \omega
$$

where $\omega$ is 0 on $\partial A$ and 1 on $\partial \Omega$.

Proof. Since $w$ is harmonic and $L(\cdot, 0)$ and $K(\cdot, 0)$ are harmonic operators, we thus can apply the alternating lemma to obtain $u, v, k$ satisfying all the above properties except possibly $k \neq 0$. To see that $k \neq 0$, we need to show $\int_{\beta} * d w \neq 0$. Clearly, $w<0$ on $\partial \Omega$, but $w \geqq 0$ on $\partial A$ by the hypothesis on $K(0,1)$ as well as the property (5) of biharmonic operators.

Let $M=\sup _{\partial A} w$. Then $M<0$. Choose any $\delta \in(M, 0)$. Let $\beta$ be the points in $A \cap \Omega$ such that $w=\delta$ there. Clearly $\int_{\beta} * d w \neq 0$. Hence $k \neq 0$ as claimed.

The following are three simple examples of biharmonic operators. 
EXAMPLE 1. Let $A$ be a compact Riemannian manifold with boundary $\partial A$. Then the Dirichlet operator is a biharmonic operator.

EXAMPLE 2. Let $\bar{R}$ be a compact Riemannian manifold with boundary $\partial \bar{R}$. Let $A^{\prime}$ be a boundary neighborhood of $\bar{R}$ with disjoint boundary components $\alpha$ and $\partial \bar{R}$. Let $\bar{B}$ be a punctured ball, disjoint from $A^{\prime}$, with deleted center $\xi$. Let $R$ be the interior of $\bar{R}\{\xi\}, A$ be $A^{\prime} \cup B$, and $\partial A$ be $\alpha \cup \partial \bar{B}$. Define $L: C(\partial A) \times C(\partial A) \rightarrow$ $H^{2}(A)$ by the following conditions:

(1) $u|\partial A=L(f)| \partial A=f=\left(f_{1} \cdot f_{2}\right)$;

(2) $u \mid B$ solves the Dirichlet problem of $B \backslash\{\zeta\}$;

(3) $\Delta u$ is a constant in $\partial \bar{R}$, chosen such that the flux condition on $\Delta u$ is satisfied;

(4) $L\left(f_{1}, 0\right)$ is a constant in $\partial \bar{R}$ chosen so that $L(\cdot, 0)$ is a harmonic operator.

(5) $L(0, \cdot)$ is a constant in $\partial \bar{R}$ so chosen that the linearity condition and condition (5) of the definition of biharmonic operators are satisfied.

Then $L$ is a biharmonic operator.

EXAMPLE 3. $L\left(f_{1}, f_{2}\right)=L_{1}\left(f_{1}\right)+\mathrm{GL}_{2}\left(f_{2}\right)$ is a biharmonic operator if $L_{1}, L_{2}$ are harmonic operator and $G$ is the Green's operator, if well defined. See [2].

3. We are now ready to prove the existence and uniqueness theorem of biharmonic principal functions which is the main result of our paper.

THEOREM. Let $A$ be a boundary neighborhood of an open Riemannian manifold $R$ with relative boundary $\partial A$. Let $L$ be a biharmonic operator on $C(\partial A) \times C(\partial A)$ and let $s$ be a biharmonic function on $A$. Then the necessary and sufficient condition for the existence of a biharmonic function $p$ in $R$ such that

$$
p-s=L(p-s, \Delta(p-s))
$$

is that the following flux condition be satisfied:

$$
\int_{\beta} * d \Delta s=0
$$

where $\beta$ is a chain homologous to $\partial A$. The function $s$ is unique up to an additive constant. Moreover, if $s$ is harmonic, then $q$ is quasiharmonic; and if $s$ is both harmonic and satisfies the flux condition $\int_{\beta} * d s=0$, then $p$ is harmonic. 
Proof. The necessity is rather routine. We will prove the sufficiency. Suppose $\int_{\beta} * d \Delta s=0$. By the existence theorem of the harmonic theory, there is a harmonic function $r$ in $R$ such that

$$
r-\Delta s=\Delta L(0, r-\Delta s) .
$$

Let $\Omega$ be a regular subregion in $R$ whose interior contains $\partial A$ and $K$ be the Dirichlet operator on $C(\partial \Omega) \times C(\partial \Omega)$ to $H^{2}(\Omega)$. By Example $1, K$ is a biharmonic operator, also $K(0,1)>0$ in the interior of $\Omega$.

We will show that $w=L(0, r-\Delta s)+s-K(0, r)$ is harmonic in $A \cap \Omega$ continuous to the boundary. Indeed, $\Delta w=\Delta L(0, r-\Delta s)+$ $\Delta s-\Delta K(0, r)=r-\Delta s+\Delta s-r=0$.

Thus, by the alternating lemma, there are functions $u, v$ and constant $k$ such that $w-(L(u, 0)+K(v, 0))=-k \omega$ where $\omega$ is harmonic on $A \cap \Omega$ with boundary data 0 on $\partial A$ and 1 on $\partial \Omega$.

There are two cases to be considered: the case that $k=0$ and otherwise.

Suppose $k=0$. We have $w-(L(u, 0)+K(v, 0))=0$. Hence $L(-u, r-\Delta s)+s=K(v, r)$ in $A \cap \Omega$. We define $p=L(-u, r-\Delta s)+s$ in $A$ and let $p=K(v, r)$ in $\Omega$. It is routine to verify that $p$ satisfies our requirement.

Suppose $k \neq 0$. Consider the function $w_{1}=L(0,1)-K(0,1)$ in $A \cap \Omega$. Clearly, $w_{1}$ is harmonic and hence, by the lemma on quasiharmonic functions in $\S 2$, there are functions $u_{1}, v_{1}$ and nonzero constant $k_{1} \neq 0$ such that $w_{1}-\left(L\left(u_{1}, 0\right)+K\left(v_{1}, 0\right)\right)=-k_{1} \omega$. Now we have $w-(L(u, 0)+K(v, 0))-k k_{1}^{-1}\left[w_{1}-\left(L\left(u_{1}, 0\right)+K\left(v_{1}, 0\right)\right)\right]=0$. We can apply the previous argument to separate $L$ and $K$ and obtain the desired $p$.

To show the uniqueness, let us suppose that there are two such functions $p_{1}$ and $p_{2}$. Since $\Delta p_{1}$ and $\Delta p_{2}$ are principal functions of $\Delta s$ for the harmonic operator $\Delta L(0, \cdot)$, they differ by a constant $k$, by the uniqueness theorem of the harmonic theory.

We claim that $k=0$. Suppose not. Let us assume that $k=$ $\Delta p_{1}-\Delta p_{2}>0$. We have a nonconstant function $p_{1}-p_{2}=p_{1}-s-$ $\left(p_{2}-s\right)=L\left(p_{1}-p_{2}, \Delta\left(p_{1}-p_{2}\right)\right)=L\left(p_{1}-p_{2}, k\right)$. Let $M=\max _{\partial A} \mid p_{1}-$ $p_{2} \mid$. We consider $p_{1}-p_{2}+M=L\left(p_{1}-p_{2}+M, 0\right)+L(0, k)$ which is nonnegative superharmonic in the entire $R$ and which has a minimum in $\partial A$ when restricted to $A$. Thus it has a minimum in the interior of $R$. This is impossible.

Therefore $\Delta\left(p_{1}-p_{2}\right)=0$. Again, consider $p_{1}-p_{2}=L\left(p_{1}-p_{2}\right.$, $\left.\Delta\left(p_{1}-p_{2}\right)\right)=L\left(p_{1}-p_{2}, 0\right)$ which when restricted to $A$ has minimum in $\partial A$. As a consequence $p_{1}-p_{2}$ has a minimum in the interior of $R$. This implies that $p_{1}-p_{2}$ is constant as required. 
Finally, suppose $s$ is harmonic, $\Delta s=0$. We may choose $r=0$. If we also have $\int_{\beta} * d s=0$, then our proof is reduced to the proof of the harmonic theory and $p$ is harmonic. If we have $\int_{\beta} * d s \neq 0$; then by adjusting a constant multiple of $L\left(0, k_{1}\right)-K\left(0, k_{1}\right)$, we see that $p$ is quasiharmonic.

As usual, we call $s$ a singularity function and $p$ a principal function of $s$ with respect to $L$.

4. In the following, we give two applications of the existence theorem. First is the construction of the singularity biharmonic functions in a closed space. The other is the construction of the biharmonic Green's function $\gamma$ for a space with boundary.

To construct the singularity biharmonic potential, let $\bar{R}$ be a closed Riemannian manifold. Choose distinct $\zeta_{0}, \zeta_{1} \in \bar{R}$ and $\Delta_{0}, \Delta_{1}$ two disjoint disks with centers $\zeta_{0}, \zeta_{1}$. Let $R=\bar{R}\left\{\zeta_{0}, \zeta_{1}\right\}, A=\Delta_{0} \cup \Delta_{1} \mid\left\{\zeta_{0}, \zeta_{1}\right\}$, $\partial A=\partial \Delta_{0} \cup \partial \Delta_{1}$. Define the singularity function $s$ so that $s$ has a positive biharmonic singularity $\zeta_{0}$ and a negative singularity near $\zeta_{1}$, i.e., $s(x) \simeq r^{2} \log r$ for $\operatorname{dim} R=2, s(x) \simeq-r$ for $\operatorname{dim} R=3, s(x) \simeq$ $-\log r$ for $\operatorname{dim} R=4$, and $s(x) \simeq r^{-n+4}$ for $\operatorname{dim} R \geqq 5$ where $n=\operatorname{dim}$ $R$ and $r=\operatorname{dist}\left(\zeta_{0}, x\right)$, the distance between $\zeta_{0}$ and $x$ for $x$ near $\zeta_{0}$. Similar remarks apply to $s(x)$ near $\zeta_{1}$ with different signs. Also, we take the flux of $\Delta s$ across $\partial \Delta_{0}$ normalized to be 1 and across $\partial \Delta_{1}$ normalized to be -1 . Hence the flux condition is satisfied. Let $L$ be the biharmonic operator corresponding to the Dirichlet operator on $\bar{A}$. By the existence theorem, there is a principal function $p$. Since $p-s=L(p-s, \Delta(p-s))$ is regular in $\bar{A}, p$ has the same singularities as $s$. We thus have a function defined in the entire space $\bar{R}$ with positive biharmonic singularity at $\zeta_{0}$ and negative biharmonic singularity at $\zeta_{1}$.

Next we construct the Green's function $\gamma$ which is characterized by the boundary data $\gamma=\Delta \gamma=0$.

Let $\bar{R}$ be a Riemannian manifold with boundary $\partial R$. Let $\zeta$ be an interior point in $\bar{R}$. We want to construct a biharmonic Green's function $\gamma(\zeta, y)$ on $\bar{R}$ which is characterized by a biharmonic singularity at $\zeta$ and boundary data $\gamma=\Delta \gamma=0$ at $\partial \bar{R}$. Choose a disk $D$ centered at $\zeta$ with boundary $\partial D$. Choose a boundary neighborhood $A^{\prime}$ of $\bar{R}$, disjoint from $D$. Let $R=\bar{R} \backslash[\zeta\} \backslash \partial \bar{R}$ and $A=A^{\prime} \cup D \backslash\{\zeta\}$. $\partial A=\partial D \cup \partial A^{\prime}$ where $\partial A^{\prime}$ is the relative boundary of $A^{\prime}$ in $R$, i.e., without $\partial \bar{R}$. Define the operator $L$ as in Example 2. The singularity function $s$ is characterized in the following way: $s(y)$ has a biharmonic singularity at $\zeta$, and $s, \Delta s$ are constant, say $c_{1}$ and $c_{2}$ at $\partial R$ with $c_{2}$ so chosen such that $\int_{\beta} * d \Delta s=0$. Since $s$ satisfies the flux condition, there is a principal function $p$ of $s$. Hence $p$ has the 
same singularity as $s$ at $\zeta$, because $p-s=L(p-s, \Delta(p-s))$ is regular in the entire $D$. By the definition of $L, p$ and $\Delta p$ are constant on $\partial R$, say equal to $c^{\prime}$ and $c^{\prime \prime}$ respectively. Then the required $\gamma=p-c^{\prime}-$ $c^{\prime \prime} \mathrm{GL}$ where GL $=\int g(x, y) d y$ is the harmonic Green's operator on 1 , which exists because $\bar{R}$ is compact bordered.

5. Our theory can be extended, by induction, to polyharmonic functions, i.e., $\Delta^{n} u=0$ for some $n$. Both the definitions and proofs will become more complicated. However, the basic idea is the same. For example, without going into details, the $n$th harmonic operator will be a linear operator $L:(C(\partial A))^{n} \rightarrow H^{n}(A)$ with conditions similar to those of the biharmonic operator.

We conclude with some open problems. The existence and uniqueness theorem is one of the three main theorems of Sario's harmonic theory. The other two theorems are the convergence theorem and the extremum theorem. A natural question for us is: what are the generalizations of these two other theorems in our theory? With these two more theorems, we will naturally consider the many applications proposed for the harmonic theory as in [3], hence a rather wide vista is in the horizon.

Acknowledgment. The author is grateful to Professor D. Hada, Professor P. A. Nickel and Professor L. Sario for valued suggestions and encouragement. Professor Nickel also developed a somewhat different theory of biharmonic principal functions, an account of which will be forthcoming. A comparison of these two theories seems interesting.

\section{REFERENCES}

1. L. Ahlfors and L. Sario, Riemann Surfaces, Princeton University Press, 1960, 382.

2. J. A. Rader, Biharmonic functions on Riemannian spaces with application to elasticity, Doctoral dissertation, UCLA, 1971, 88.

3. B. Rodin and L. Sario, Principal Functions, Van Nostrand (now Springer Verlag), 1968, 347 .

Received September 11, 1978.

North Carolina State University

RELAIGH, NC 27650 



\title{
PACIFIC JOURNAL OF MATHEMATICS
}

\section{EDITORS}

\author{
DoNALD BABBITT (Managing Editor) \\ University of California \\ Los Angeles, CA 90024 \\ HUGo RossI \\ University of Utah \\ Salt Lake City, UT 84112 \\ C. C. MOORE and ANDREW OGG \\ University of California \\ Berkeley, CA 94720
}

J. DugundJI

Department of Mathematics

University of Southern California

Los Angeles, CA 90007

R. FINN and J. Milgram

Stanford University

Stanford, CA 94305

\section{ASSOCIATE EDITORS}
E. F. BeCKenbach
B. H. NeumanN
F. WOLF
K. YoshidA

\section{SUPPORTING INSTITUTIONS}

\author{
UNIVERSITY OF BRITISH COLUMBIA \\ CALIFORNIA INSTITUTE OF TECHNOLOGY \\ UNIVERSITY OF CALIFORNIA \\ MONTANA STATE UNIVERSITY \\ UNIVERSITY OF NEVADA, RENO \\ NEW MEXICO STATE UNIVERSITY \\ OREGON STATE UNIVERSITY \\ UNIVERSITY OF OREGON
}

\author{
UNIVERSITY OF SOUTHERN CALIFORNIA \\ STANFORD UNIVERSITY \\ UNIVERSITY OF HAWAII \\ UNIVERSITY OF TOKYO \\ UNIVERSITY OF UTAH \\ WASHINGTON STATE UNIVERSITY \\ UNIVERSITY OF WASHINGTON
}

The Supporting Institutions listed above contribute to the cost of publication of this Journal, but they are not owners or publishers and have no responsibility for its content or policies.

Mathematical papers intended for publication in the Pacific Journal of Mathematics should be in typed form or offset-reproduced, (not dittoed), double spaced with large margins. Please do not use built up fractions in the text of the manuscript. However, you may use them in the displayed equations. Underline Greek letters in red, German in green, and script in blue. The first paragraph or two must be capable of being used separately as a synopsis of the entire paper. Please propose a heading for the odd numbered pages of less than 35 characters. Manuscripts, in triplicate, may be sent to any one of the editors. Please classify according to the scheme of Math. Reviews, Index to Vol. 39. Supply name and address of author to whom proofs should be sent. All other communications should be addressed to the managing editor, or Elaine Barth, University of California, Los Angeles, California, 90024.

50 reprints to each author are provided free for each article, only if page charges have been substantially paid. Additional copies may be obtained at cost in multiples of 50 .

The Pacific Journal of Mathematics is issued monthly as of January 1966. Regular subscription rate: $\$ 84.00$ a year $(6$ Vols., 12 issues). Special rato: $\$ 42.00$ a year to individual members of supporting institutions.

Subscriptions, orders for numbers issued in the last three calendar years, and changes of address shoud be sent to Pacific Journal of Mathematics, P.O. Box 969, Carmel Valley, CA 93924, U.S.A Old back numbers obtainable from Kraus Periodicals Co., Route 100, Millwood, NY 10546.

\section{PUBLISHED BY PACIFIC JOURNAL OF MATHEMATICS, A NON-PROFIT CORPORATION}

Printed at Kokusai Bunken Insatsusha (International Academic Printing Co., Ltd.). 8-8, 3-chome, Takadanobaba, Shinjuku-ku, Tokyo 160, Japan. 


\section{Pacific Journal of Mathematics}

\section{Vol. 86, No. 2 December, 1980}

Graham Donald Allen, David Alan Legg and Joseph Dinneen Ward, Hermitian

liftings in Orlicz sequence spaces ............................... 379

George Bachman and Alan Sultan, On regular extensions of measures ........ 389

Bruce Alan Barnes, Representations Naimark-related to $*$-representations; a

correction: "When is a representation of a Banach $*$-algebra

Naimark-related to a $*$-representation?" ........................ 397

Earl Robert Berkson, One-parameter semigroups of isometries into $H^{p} \ldots \ldots .403$

M. Brodmann, Piecewise catenarian and going between rings ............ 415

Joe Peter Buhler, A note on tamely ramified polynomials ............... 421

William Lee Bynum, Normal structure coefficients for Banach spaces ........ 427

Lung O. Chung, Biharmonic and polyharmonic principal functions ......... 437

Vladimir Drobot and S. McDonald, Approximation properties of polynomials

with bounded integer coefficients .............................

Giora Dula and Elyahu Katz, Recursion formulas for the homology of

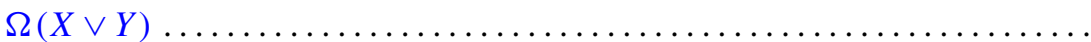

John A. Ernest, The computation of the generalized spectrum of certain Toeplitz operators ...................................... 463

Kenneth R. Goodearl and Thomas Benny Rushing, Direct limit groups and the

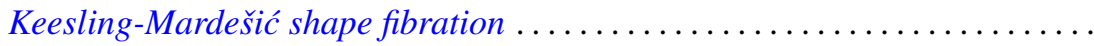

Raymond Heitmann and Stephen Joseph McAdam, Good chains with bad contractions

Patricia Jones and Steve Chong Hong Ligh, Finite hereditary near-ring-semigroups .

Yoshikazu Katayama, Isomorphisms of the Fourier algebras in crossed

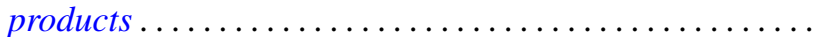

Meir Katchalski and Andrew Chiang-Fung Liu, Symmetric twins and common transversals. .

Mohammad Ahmad Khan, Chain conditions on subgroups of LCA groups ....

Helmut Kröger, Padé approximants on Banach space operator equations ...

Gabriel Michael Miller Obi, An algebraic extension of the Lax-Milgram theorem . .

S. G. Pandit, Differential systems with impulsive perturbation .

Richard Pell, Support point functions and the Loewner variation ...

J. Hyam Rubinstein, Dehn's lemma and handle decompositions of some

4-manifolds

James Eugene Shirey, On the theorem of Helley concerning finite-dimensional subspaces of a dual space.

Oved Shisha, Tchebycheff systems and best partial bases.... 